\title{
DISCOVERY OF INTERSTELLAR CIRCULAR POLARIZATION IN THE DIRECTION OF THE CRAB NEBULA
}

\author{
P. G. MARTIN* \\ Institute of Theoretical Astronomy, Madingley Road, Cambridge, England \\ and \\ R. ILLING and J. R. P. ANGEL \\ Columbia Astrophysics Laboratory, Columbia University, New York, N.Y. 10027, U.S.A.
}

\begin{abstract}
A search in many small regions of the Crab Nebula has resulted in the detection of a small component of circular polarization. The variation of the sign and magnitude with position in the Nebula indicates that the polarization is of interstellar origin. On the basis of the polarity, strength, and colour dependence, it is concluded that the composition of the aligned grains causing this polarization is dielectric. Metallic particles are clearly ruled out.
\end{abstract}

\section{Introduction}

This paper reports the detection of circular polarization in the Crab Nebula, interpreted as due to interstellar grains. Since the theoretical discussion which motivated these observations appears in the preceding paper (Martin, 1972, Paper I) it will be summarized only briefly here. The presence of aligned anisotropic (elongated) grains makes the interstellar medium not only linearly dichroic, as observations of the interstellar linear polarization indicate, but also linearly birefringent. Therefore, when linearly polarized radiation passes through the medium, a component of circular polarization is produced if the position angle of this incident polarization does not coincide with either principal axis of the medium. The resulting maximum ellipticity (ratio of ellipse axes) is roughly equal to the fraction of linear polarization $P$ caused by the interstellar medium. Circular polarization from interstellar grains is of interest because, as shown in Paper I, the magnitude and sign vary with wavelength in a manner characteristic of the grain material, offering an opportunity to distinguish clearly between dielectric and metallic grains. Restrictions placed on the composition of the grains by our observations are discussed below.

An almost ideal source for the study of interstellar birefringence is the Crab Nebula. The strongly polarized synchrotron continuum is seen through a foreground interstellar medium whose polarization properties are known independently from stellar observations. The range of position angles of linear polarization in the Nebula allows a check of the angular dependence of the circular component, for it can be assumed that the optical properties of the interstellar medium are virtually constant over the small angular size of the Nebula. The variation of circular polarization across the Nebula is important in establishing that the observed effect is really interstellar. Rees

* Present address: Dept. of Astronomy, University of Toronto, Toronto, Ontario, Canada. 
(1971) has predicted that the radiation will be intrinsically circularly polarized if the nebular magnetic field is $30-\mathrm{Hz}$ electromagnetic radiation rather than a DC field; however, this effect can be distinguished by the predicted variation with position, which is quite different for the two mechanisms.

\section{Observations of the Crab Nebula}

\subsection{EARLY OBSERVATIONS}

In the past, observations of the Crab Nebula have failed to show circular polarization above the limits of their observational accuracy. Using a photographic method, Oetken (1966a) found values around $2 \%$, but these were questioned after new photoelectric measurements were taken (Oetken, 1966b). In this latter paper, it is reported that Serkowski also had found no significant polarization using a $96^{\prime \prime}$ diaphragm, the Stokes parameter $V$ being $0.22 \pm 0.5 \%$. Although not looking for this particular effect, Wolstencroft (1966) obtained some limits with a $40^{\prime \prime}$ diaphragm, $V=0.2 \pm 0.65 \%$ at visual wavelengths and $0.15 \pm 0.5 \%$ in the red. The only measurements of sufficient accuracy to detect this effect were made by Landstreet and Angel (1971) when investigating the predictions of the 'synchro-Compton' emission process(Rees, 1971). For two 7-s spots in the Nebula in the wavelength region 4000-5800 $\AA$, they found $V=-0.024 \pm 0.040$ and $-0.034 \pm 0.047 \%$. These latter results are discussed below along with the new measurements.

\subsection{INTERSTELLAR AND INTRINSIC LINEAR POLARIZATION}

The interstellar linear polarization can be estimated by studying stars in the vicinity of the Nebula. However, there are only three stars in the catalogues of Hiltner (1956) and Hall (1958) within a few degrees and at nearly the same distance. The star HD 36879 shows $2 \%$ linear polarization at position angle $152^{\circ}$, HD $36547,1.4 \%$ at $149^{\circ}$, and HDE $245310,3.9 \%$ at $146^{\circ}$. To supplement these data, Cocke et al. (1970) have measured the polarization of 10 stars fainter than twelfth magnitude within a few minutes of arc of the Nebula. However, no distances are known. If these stars are main sequence stars as far away as the $\mathrm{Crab}$, they would have spectral types about $\mathrm{B}_{3}$ and later. A mean polarization $2.0 \pm 0.2 \%$ at $147^{\circ} \pm 3^{\circ}$ is given, but there was a larger scatter of about $10^{\circ}$ in the position angles (Gehrels, private communication).

Observations of the Crab Nebula pulsar NP0532 could possibly give another determination of the interstellar polarization. It is found that near the intensity peaks of both the main and secondary pulses, the linear polarization decays to nearly zero. Kristian et al. (1970) suggest that the residual of about $2 \%$ at $160^{\circ}$ is largely of interstellar origin. Observations of the secondary pulse by Cocke et al. (1970) are consistent with this interpretation, but there is a disagreement of about $40^{\circ}$ in the position angle at the polarization minimum of the main pulse.

Maps of the linear polarization over the face of the Nebula have been made by Walraven (1957), Woltjer (1957), and Hiltner (1957). No new measurements of the linear polarization were made during this work, and we have adopted Woltjer's 
measurements for the wavelength range 5200-6400 $\AA$ (Plate I). For the regions we have observed, the estimated uncertainty in the polarization is $2-3 \%$, and $4^{\circ}$ to $6^{\circ}$ in position angle. Removal of the small component of interstellar polarization gives the intrinsic linear polarization used in our analysis.

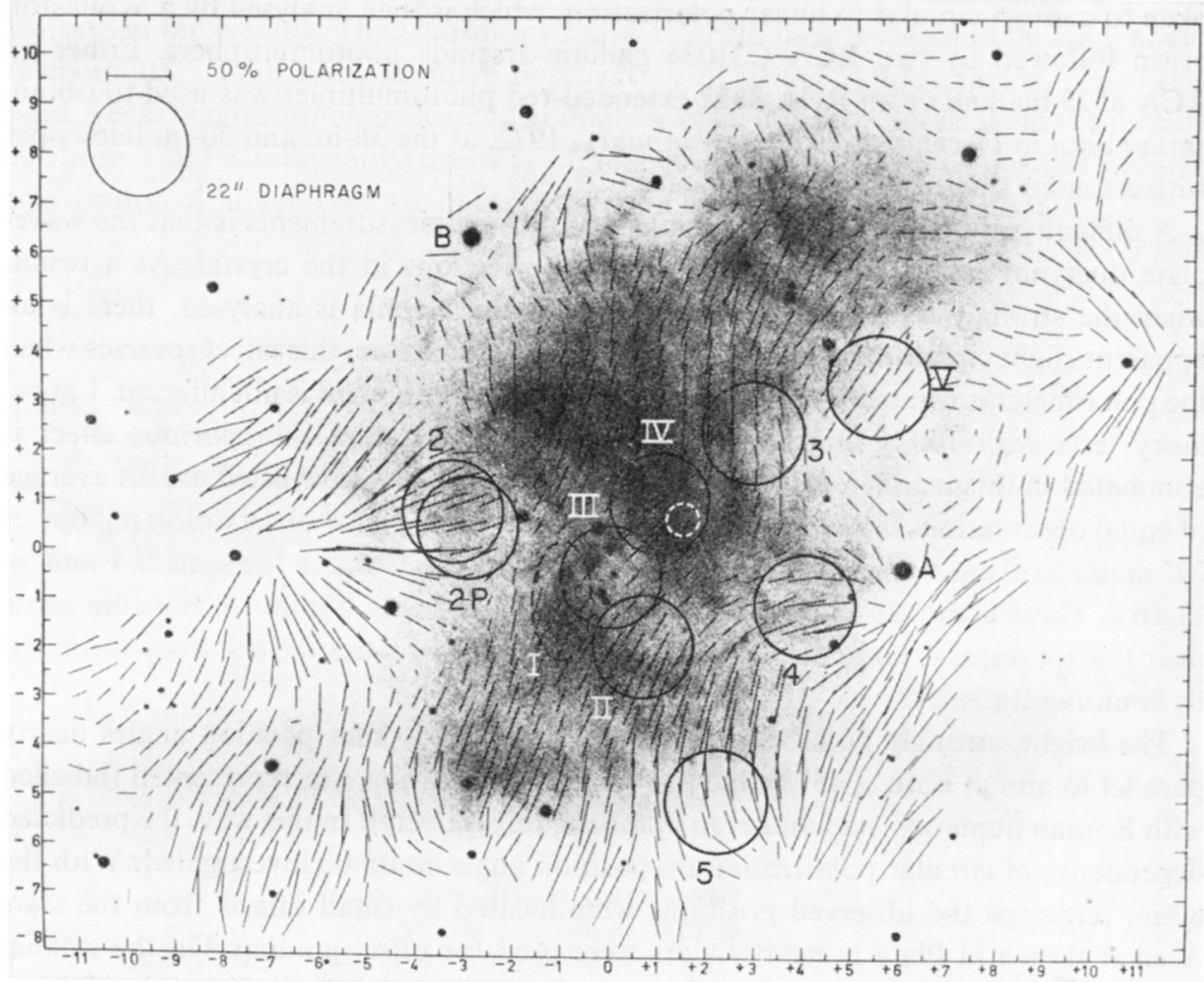

Plate I. A map of linear polarization over the face of the Crab Nebula (from Woltjer, 1957), showing the positions chosen to search for circular polarization. Dashed regions were examined previously by Landstreet and Angel (1971).

It is assumed on the bais of synchrotron emission that the intrinsic polarization is the same at all wavelengths. No Faraday rotation occurs at optical frequencies(Conway, 1971). However, there exists the possibility that in regions with strong filaments the percentage polarization will vary with colour if the contribution to the total radiation from the unpolarized emission lines is important. The dominant lines are from [O II] in the blue, $[\mathrm{O} \mathrm{III}]$ in the yellow, and $\mathrm{H} \alpha+[\mathrm{N} \mathrm{II}]$ and $[\mathrm{S} \mathrm{II}]$ in the red. Of the positions actually observed only two (those labelled 2 and 4 ) could be affected; with the relevant line and continuum intensities measured by Woltjer (1958), we have calculated that there might be a $20 \%$ dilution from the lines. Only position III could be contaminated with starlight. No allowances have been made for these possibilities. 


\subsection{INSTRUMENTATION AND OBSERVATIONS}

The polarimeter described previously by Angel and Landstreet (1970) and Landstreet and Angel (1972) was used to make observations of circular polarization, mostly at the Cassegrain focus of the 82-in. telescope of McDonald Observatory in February, 1972. This instrument uses a Pockels cell acting as an electrically switched quarter-wave plate to convert circular to linear polarization, which is then analysed by a Wollaston prism followed by two RCA C31034 gallium-arsenide photomultipliers. Either an RCA 8575 bialkali or an RCA 8852 extended-red photomultipler was used to obtain earlier data in December, 1971, and January, 1972, at the 36-in. and 30-in. telescopes with a similar single-channel polarimeter.

A difficulty of the Pockels-cell instrument for these measurements is that the waveplate does not reverse exactly because of imperfections in the crystal. As a result, when the strongly linearly polarized light from the Nebula is analysed, there is an apparent slight circular polarization of up to $0.3 \%$. However, this effect reverses when the polarimeter is rotated $90^{\circ}$, while the true circular component is not affected. Laboratory tests with $100 \%$ linearly polarized light confirm that the spurious effect is eliminated in this manner. In every case the results that we have listed are an average of equal observations taken at $90^{\circ}$ orientations. We selected for observation regions of $22^{\prime \prime}$ diameter from Woltjer's (1957) map, shown in Plate I, where the scale is 1 unit = $11.16 \mathrm{~s}$. These areas are as large as possible consistent with a uniform position angle over the aperture. A large diaphragm size is desirable because the accuracy is limited by counting statistics.

The bright, strongly polarized regions were chosen so that position angles nearly parallel to and at both $\pm 45^{\circ}$ to the interstellar polarization are represented (labelled with Roman numerals, odd and even numbers, respectively). In this way, the predicted dependence of circular polarization on position angle could be investigated. With the 82-in. telescope the observed positions were located by small offsets from the stars A en B shown in Plate I; nearby stars were used for offset guiding. On the smaller telescopes much larger offsets had to be made relative to a more distant guide star, BD $22^{\circ} 947$, because stars A and B were visible only during the best seeing.

\subsection{ObSERVATIONAL RESUlTS AND DISCUSSION}

The circular polarization measurements, expressed as percentages, are given in Table I for the four different wavelength regions studied (characteristically infrared, broad red, yellow, and blue). The standard deviations quoted are calculated from counting statistics alone. For each colour separate tabulations for the same position in the Nebula represent an observation on a different night. The values are corrected for the night sky background (which was found to be unpolarized), and a small correction has been applied for instrumental efficiency. The sign convention adopted is the same as in Paper I: the Stokes parameter $V$ is positive (right handed) when, to an observer facing the Nebula, the electric vector in a fixed plane rotates counterclockwise. Observations of the circularly polarized white dwarfs Grw $+70^{\circ} 8247$ and G99-37 
were made to establish the sense of polarization. The linear polarization listed in Table $I$ is the vector-averaged polarization from Woltjer (1957) corrected for interstellar polarization.

The measurements in Table I show small but definite polarization in the broad red and infra-red bands, clearly changing sing from one region to another. It is seen that the measured effect depends on the position angle of the linear polarization and not on position in the Nebula. Thus, positions 2 and 4, which have a common position angle of about $100^{\circ}$, both show negative polarization of about $-0.1 \%$ in the broad red band. These two positions are located at diametrically opposite points from the pulsar. Positions 3 and 5 , both with a linear position angle of about $10^{\circ}$, show positive circular polarization although they are in quite different directions from the pulsar.

TABLE I

Observations of circular polarization in the Crab Nebula

\begin{tabular}{|c|c|c|c|c|c|c|}
\hline \multirow[t]{2}{*}{ Position } & \multirow{2}{*}{\multicolumn{2}{|c|}{$\begin{array}{l}\text { Linear } \\
\text { polarization } \\
\mathscr{P}(\%) \quad \theta\left({ }^{\circ}\right) \text { (p.a.) }\end{array}$}} & \multirow{2}{*}{$\begin{array}{l}\text { Infra-red } \\
8800-7800 \AA\end{array}$} & \multicolumn{3}{|c|}{ Circular polarization $(\%)$} \\
\hline & & & & $\begin{array}{l}\text { Broad red } \\
8800-6000 \AA\end{array}$ & $\begin{array}{l}\text { Yellow } \\
5600-4200 \AA\end{array}$ & $\begin{array}{l}\text { Blue } \\
4700-3500 \AA\end{array}$ \\
\hline 3 & 17.3 & 6.5 & $\begin{array}{l}+0.235 \div 0.092 \\
+0.147 \div 0.037\end{array}$ & $\begin{array}{l}+0.110 \pm 0.027 \\
+0.142 \pm 0.045 \\
+0.109 \pm 0.038\end{array}$ & $+0.09 \pm 0.06$ & $\begin{array}{l}+0.05 \div 0.15^{\mathrm{b}} \\
-0.08 \div 0.06^{\mathrm{a}}\end{array}$ \\
\hline 5 & 32.2 & 15.9 & & $+0.280 \pm 0.040$ & & \\
\hline 4 & 32.1 & 100.1 & $-0.201 \pm 0.062$ & $\begin{array}{r}+0.29 \pm 0.11^{\mathrm{a}} \\
-0.33 \pm 0.13^{\mathrm{a}} \\
-0.110 \pm 0.038 \\
-0.061 \pm 0.024\end{array}$ & & $\begin{array}{l}-0.14 \pm 0.10^{\mathrm{a}} \\
-0.08 \pm 0.07^{\mathrm{a}} \\
+0.14 \pm 0.13^{\mathrm{a}}\end{array}$ \\
\hline 2 & 38.9 & 108.8 & & $-0.142 \pm 0.033$ & & \\
\hline $2 \mathbf{P}$ & 30.6 & 103.3 & & & $-0.08 \pm 0.06^{b}$ & \\
\hline II & 31.4 & 151.5 & $\begin{array}{l}0.2350 .092 \\
-0.152 \pm 0.051\end{array}$ & $\begin{array}{l}-0.099 \pm 0.021 \\
-0.017 \pm 0.048 \\
-0.053 \pm 0.032\end{array}$ & & \\
\hline III & 18.9 & 138.9 & $-0.105 \pm 0.052$ & $-0.016 \pm 0.023$ & $-0.02+0.04$ & \\
\hline IV & 21.6 & 141.6 & $-0.239 \pm 0.115$ & $-0.047 \pm 0.039$ & $-0.024+0.040^{c}$ & \\
\hline V & 25.6 & 145.9 & & $+0.063 \pm 0.092$ & $-0.034 \pm 0.047^{c}$ & \\
\hline I & 16.9 & 155.3 & & & & \\
\hline
\end{tabular}

All observations with the 82-in. telescope except (a) 30-in., and (b) 36-in.

(c) From Landstreet and Angel (1971).

The observed dependence of circular polarization on position angle is characteristic of transmission through a wave plate or birefringent medium and is not consistent with an interpretation in terms of intrinsic circular polarization of the Nebula predicted by the 'synchro-Compton' theory. In this theory the polarization would be strongest along the rotation axis of the pulsar, taken to be the axis of the observed linear polarization, and of opposite sign at apposite points from the pulsar. Furthermore, this theory predicts a comparatively large effect $(1 \%)$ independent of wavelength which is also in contradiction with the observations. Thus, the new data add weight to the 
conclusion, already drawn from the absence of circular polarization in visible light (Landstreet and Angel, 1971), that 'synchro-Compton' emission cannot contribute more than a few per cent of the light of the Nebula.

We are also confident that the observed effect is not instrumental for the following reasons. First, the measurements of each position are generally reproducible and show a scatter consistent with the calculated error from counting statistics. This is despite changes of telescope and of polarimeter, and repeated realignments of the optics. The only discrepancy is in the broad red data for position 4 taken with two different telescopes. This might possibly be explained by a slight offset error on the smaller telescope which would mean a different region was being measured. While the rotation cancels all spurious effects in the polarimeter, there is the remote possibility that both telescope mirror systems were acting as wave plates with the same fast and slow axis positions, which would result in the measured dependence of polarization on position angle. Against this we can argue that any wave-plate effect would probably result in a greater polarization at shorter wavelengths, opposite to what is observed. Also, at least for the 82 -in. telescope which is symmetrically aluminized for minimum linear polarization (about $0.1 \%$ ), there is no reason to expect birefringence.*

Circular polarization from interstellar grains is expected to change for different regions of the Nebula according to

$$
V \mid \mathscr{P}=A(\lambda) \sin 2\left(\theta-\theta_{0}\right),
$$

where $\mathscr{P}$ is the intrinsic linear polarization at position angle $\theta$ (Table I), $\theta_{0}$ is the position angle of the interstellar polarization, and $A(\lambda)$ is the retardation which depends on the number of grains and on the grain material. By plotting $V / \mathscr{P}$ (which is very nearly equal to twice the ellipticity) against $\theta$, we should be able to fit a sine curve to determine $\theta_{0}$ and the sign and magnitude of $A(\lambda)$.

Figure 1 displays our results in this manner. Separate measurements of the same point on different nights are shown individually to indicate the repeatability and have been separated slightly in position angle for clarity. Several symbols are used to distinguish between different parts of the Nebula when the position angles are nearly the same. For each colour the best fitting values of $A$ and $\theta_{0}$ were determined by a least squares fitting procedure. The corresponding functions $A(\lambda) \sin 2\left(\theta-\theta_{0}\right)$ are plotted.

We make the following observations. First, in the infra-red and yellow the data points from many different regions in the Nebula are very well fitted by a sine curve. The same trend holds in the broad red, but there appear to be some systematic errors in addition to what can be attributed to counting statistics. The fit would be improved if, as mentioned previously, the measured values in positions 2 and 4 were low because of filamentary light. In the blue the poor quality data do not warrant the drawing of the best fit curve; the formal solution has a large amplitude (2), and passes through

* The circular polarization measurements have been confirmed by Martin and Angel using a third telescope, the 84-in. at Kitt Peak National Observatory. 


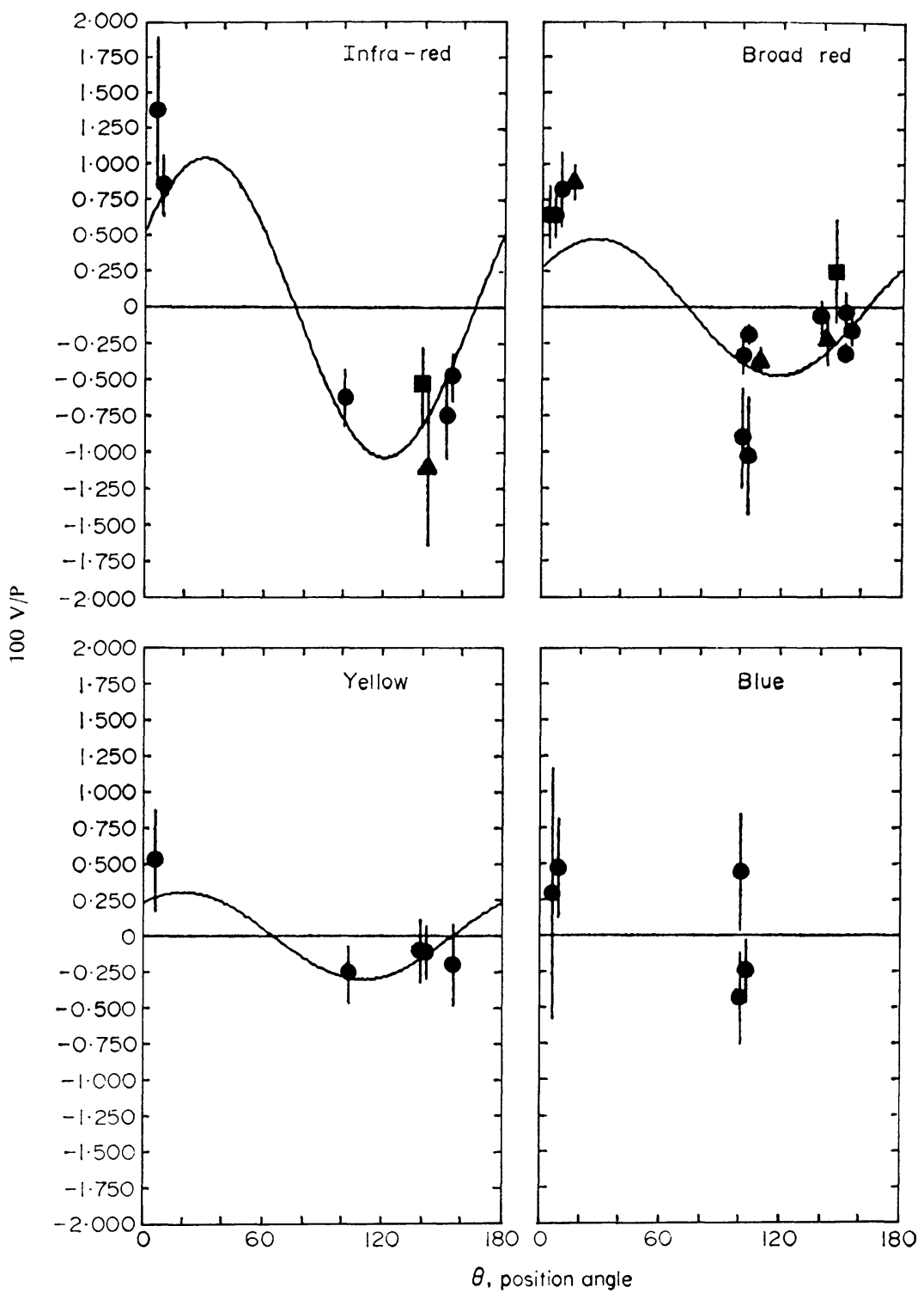

Fig. 1. Measured values of the normalized circular polarization, $V / \mathscr{P}$, vs the position angle of the intrinsic linear polarization. Curves have been fitted by a least-squares procedure.

zero near the two groups of data points. The linear dependence of $V$ on $\mathscr{P}$ is illustrated by the agreement of the broad red values of $V / \mathscr{P}$ for positions 3 and 5 , which have nearly the same position angle but $\mathscr{P}$ different by a factor 2 .

Seondly, the values of $\theta_{0}$ obtained for each colour are consistent with each other, the best fit angles being 165,162 , and $155^{\circ}$ for the infra-red, broad red and yellow data respectively, with a weighted average of $163^{\circ}$. The close agreement found is likely 


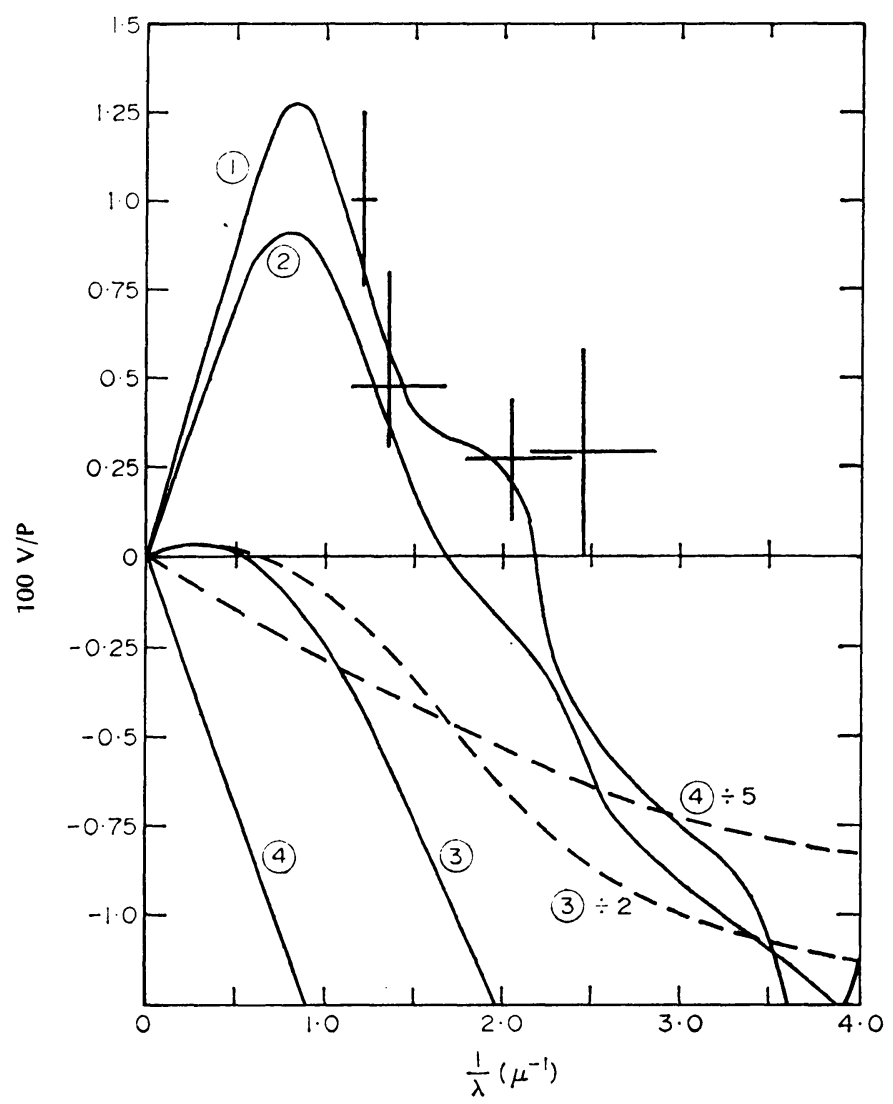

Fig. 2. Comparison of the observed circular polarization with that expected for different grain 'materials': $1 \mathrm{~m}=1.5,2 \mathrm{~m}=1.5-0.1 i, 3 \mathrm{~m}=1.5-0.5 i, 4 \mathrm{~m}=1.5-1.5 i$. The observations clearly indicate dielectric rather than metallic grains.

fortuitous; from fitting the curves for the individual colours we estimate the uncertainty in $\theta_{0}$ to be about $10^{\circ}$. The interstellar grain theory predicts that $\theta_{0}$ should be the same as the position angle given by the interstellar linear polarization, found above to be in the range $145-160^{\circ}$. Thus there is good agreement, within the expected errors, for the direction of the grain orientation deduced independently from the interstellar linear and circular polarization measurements.* Thirdly, $A(\lambda)$ decreases with decreasing wavelength, although the functional dependence is uncertain. This colour dependence holds point by point in the Nebula.**

* If the position angle of the interstellar polarization were not known, there would be an ambiguity in $\theta_{0}$ of $90^{\circ}$, and in the sign of the amplitudes. Here the good agreement of the two determinations removes the ambiguity.

** It is now clear why interstellar circular polarization was not found in the earlier measurements (Landstreet and Angel, 1971); for the regions studied the position angles were nearly parallel to the interstellar direction, and in any case the effect is small in the yellow wave band. 
The measured sign and amplitudes of $A(\lambda)$ can be compared with the theoretical predictions for different complex refractive indices to derive valuable information about the grain material. In Paper I it is shown how the relative amounts of linear and circular polarization for a given grain model can be calculated from the Mie theory for infinite cylinders. Figure 2 in Paper I shows the relative amounts of circular polarization expected from different grain materials when the linear polarization from each grain model has been normalized to the same value. In this calculation the linear polarization observations have been fitted first, because the same grains must be responsible for both the linear and circular polarization. The proper wavelength scale is found by choosing the appropriate grain size for each complex refractive index to obtain a match with the mean observed wavelength dependence of interstellar linear polarization. As shown in Figure 1 of Paper I this fit can be obtained for a large variety of grain materials, leaving a large ambiguity in this respect in the interpretation of the observations. However, the circular polarization is very dependent on composition.

Figure 2 of this paper shows the predicted values of $A(\lambda)$ for the Crab Nebula for different grain compositions; ${ }^{*}$ the scale is determined from Equations (4) and (5) of Paper I assuming the visual interstellar linear polarization to be $2 \%$. This procedure essentially eliminates the number of grains along the line of sight, because it enters equally in the equations describing the degree of circular and linear polarization from the medium. For Figure 2 we have calculated the amplitudes which best fit our observational results, assuming $\theta_{0}$ to be $163^{\circ}$. The vertical bars show the estimated uncertainty.

The observed values of $A(\lambda)$ agree well with the predicted curve for pure dielectric grains. The sign, strength, and general trend with wavelength all support this interpretation. On the basis of curve 2, drawn for a refractive index $1.5-0.1 i$, it appears that the imaginary part of the refractive index does not exceed 0.1 . As discussed in Paper I, some materials which fall in this category are 'ices', including 'dirty ice', silicates, and silicon carbide. Aligned metallic particles, such as graphite or iron, can be ruled out by the difference in sign of the polarization from that predicted and by the conspicuous absence of strong polarization in the blue, which would be expected to be in excess of $0.5 \% * *$.

\section{Conclusion}

The circular polarization detected in the Crab Nebula shows all the characteristic features expected of polarization by aligned interstellar grains. The dependence of the

* The curves shown display the general trend as the imaginary part of the complex refractive index increases from zero. Details such as the exact wavelength of the crossing for dielectric grains depend on the size distribution, the degree of alignment, and the variations of the refractive index in the optical region of the spectrum.

** However, we cannot exclude spherical or unaligned metallic particles, or small particles which would not contribute to the polarization (and extinction) at visible wavelengths. 
circular component on the linear polarization position angle rather than position in the Nebula indicates an interstellar origin. The sign, strength, and wavelength dependence of the effect all indicate that, at least along a 2-kpc path in the Galactic plane towards the anticentre, the composition of the aligned grains causing the polarization is dielectric rather than metallic. Since the interstellar grain composition may vary in different parts of the Galaxy, it would be desirable to observed more objects in other directions.

In view of the important conclusions to be derived from circular polarization measurements, our observations of the Crab Nebula will be extended to cover more position angles and wavelength bands and will include direct measurement of the linear polarization in each region and colour. Better information about the interstellar linear polarization can be obtained by using narrow-band filters centred on the strongest emission lines of the filamentary structure. This will give the linear polarization directly in front of the Nebula and will also show any changes that might occur across the Nebula. With these improvements it is hoped that accurate curves of the type shown in Figure 1 can be constructed to give detailed information about the wavelength dependence of circular polarization for comparison with theoretical models.

\section{Acknowledgements}

It is a pleasure to acknowledge the cooperation of Prof B. Warner and to thank the Director of McDonald Observatory for making the 82-in., 36-in., and 30-in. telescopes available to us. This work was supported by the Research Corporation, the National Science Foundation under grant GP 31356X, and the National Aeronautics and Space Administration under grant NGR 33-008-102. P.G.M. is grateful for financial assistance from Commission 38 of the IAU and from Churchill College, Cambridge. This is Columbia Astrophysics Laboratory Contribution No. 63. J.R.P.A. is an Alfred P. Sloan Research Fellow. This is a part of work published previously (Martin et al., 1972).

\section{References}

Angel, J. R. P. and Landstreet, J. D.: 1970, Astrophys. J. Letters 162, L61.

Cocke, W. J., Disney, M. J., Mancaster, G. W., and Gehrels, T., 1970, Natures 227, 1327.

Conway, R. G.: 1971, in R. D. Davies and F. G. Smith (eds.), 'The Crab Nebula', IAU Symp. 46, 292.

Hall, J. S.: 1958, Publ. U.S. Naval Obs. 17, 275.

Hiltner, W. A.: 1956, Astrophys. J. Suppl. 2, 389.

Hiltner, W. A.: 1957, Astrophys. J. 125, 300.

Kristian, J., Visvanathan, N., Westphal, J. A., and Snellen, G. H.: 1970, Astrophys. J. 162, 475.

Landstreet, J. D. and Angel, J. R. P.: 1971, Nature 230, 103.

Landstreet, J. D. and Angel, J. R. P.: 1972, Astrophys. J. Letters 174, L127.

Martin, P. G.: 1972. Monthly Notices Roy Astron. Soc. 159, 179, (Paper I).

Martin, P. G., Illing, R., and Angel, J. R. P.: 1972, Monthly Notices Roy. Astron. Soc. 159, 191.

Oetken, L.: 1966a, Astron. Nachr. 289, 13. 
Oetken, L.: 1966b, Astron. Nachr. 289, 189.

Rees, M. J.: 1971, in R. D. Davies and F. G. Smith (eds.), 'The Crab Nebula', IAU Symp. 46, 407.

Walraven, Th.: 1957, Bull. Astron. Inst. Neth. 13, 293.

Wolstencroft, R.: 1966, Observatory, 86, 223.

Woltjer, L.: 1957, Bull. Astron. Inst. Neth. 13, 301.

Woltjer, L.: 1958, Bull. Sstron. Inst. Neth. 14, 39. 